\title{
Delayed Diagnosis of Atypical Presentation of Myasthenia Gravis
}

\author{
Moneeb Sefeldawla Mustafa, Megan Marshal, Emer Ahern, Paul Crowley \\ Internal Medicine Department, St. Luke General Hospital, Kilkenny, Ireland
}

Received: 14/11/2017

Accepted: 20/11/2017

Published: 21/12/2017

How to cite this article: Mustafa MS, Marshal M, Ahern E, Crowley P. Delayed diagnosis of atypical presentation of myastenia gravis. EJCRIM 2017;4: doi:10.12890/2017_000785.

Conflicts of Interests: The Authors declare that there are no competing interests.

This article is licensed under a Commons Attribution Non-Commercial 4.0 License

\section{ABSTRACT}

Myasthenia gravis is an autoimmune disorder affecting the neuromuscular junction, resulting in muscle fatiguability and weakness.

The pathological characteristics of the disorder include ocular weakness resulting in diplopia and/or ptosis. More generally, the disease can result in fluctuant weakness of skeletal muscle, predominantly affecting ocular, bulbar and respiratory muscles. Autoimmunity in this instance is mediated by IgG anti-acetylcholine receptor antibodies that results in an impaired structure of postsynaptic neurotransmission. Approximately $15 \%$ of patients with myasthenia gravis present with bulbar symptoms, of which isolated bulbar symptoms are seen only on occasion. As with our patient, this presentation is most commonly seen in men with late-onset myasthenia gravis.

We present a case of an 83-year-old male who presented with a 1 year history of dysphagia for solids and fatigable dysarthria. Following a diagnosis of myasthenia gravis, he was initiated on corticosteroid treatment. He later descended into myasthenic crisis, requiring invasive ventilation measures due to a failure of both non-invasive ventilation and intravenous immunoglobulin G (IVIG) to achieve therapeutic goals.

\section{LEARNING POINTS}

- Isolated bulbar symptoms in an elderly man warrants consideration of myasthenia gravis to be included in the differential diagnosis.

- There is possibly an increased risk of myasthenic crisis in late-onset isolated bulbar myasthenia gravis.

- Empirical treatment for myasthenia gravis must be administered within a hospital setting, paying attention to respiratory tract infection and corticosteroid treatment as triggers for myasthenic crisis.

\section{KEYWORDS}

Glanzmann thrombasthenia, bleeding, rFVIIa

\section{CASE DESCRIPTION}

An 83-year-old male presented with a 1 year history of dysphagia for solids that intensified over a 2 week period. The patient also complained of fatigable dysarthria that worsened throughout the day, and on presentation he had slurred speech.

On examination, no ptosis or diplopia was elicited and patient denied history of same. Past medical history included hypertension, hypercholesterolaemia, and benign prostatic hyperplasia. Differential diagnoses at this point included amyotrophic lateral sclerosis, cerebrovascular accident, paraneoplastic disease, and oesophageal motility disorder. CT brain, neck and thorax were performed and determined to be normal. Barium swallow revealed an incoordination of swallow of the pharynx with some leakage of contrast into the oesophagus. Following neurology review, a diagnosis of myasthenia gravis was made and treatment was initiated, consisting of pyridostigmine, prednisone $500 \mathrm{mg}$ per day for 3 days, with an ongoing dose of $25 \mathrm{mg}$. 
One day post treatment, the patient reported that his speech and swallow had improved. However, 4 days post treatment, the patient developed type II respiratory failure with hypoxia, hypercapnia and decreased air entry in basal segments, bilaterally, along with left bundle branch block on ECG and chest X-ray showing right-sided infiltrate.

The patient was treated with tazobactam/piperacillin and clarithromycin for aspiration pneumonia. This treatment elicited minimal improvement overnight. Following this, the patient was assessed by the neurology team who suggested a diagnosis of myasthenic crisis secondary to pneumonia and prednisone treatment. The patient was treated for myasthenic crisis with neostigmine and glycopyrronium bromide and was initiated on non-invasive ventilation.

Failure of this treatment led to the patient commencing intravenous immunoglobulin G (IVIG) therapy and was continued on this for 1 day. Failure of these measures to achieve adequate respiratory function prompted a case management review with the senior consultant in intensive care and elective invasive ventilation was initiated, while continuing IVIG therapy for a total of 5 days.

Following 4 days in the intensive care setting, the patient was extubated and discharged to the ward.

His medical treatment continued with pyridostigmine and he was subsequently recommenced on prednisone 25 mg following control of myasthenia symptoms. The patient was discharged from hospital to be reviewed in the outpatient department in 4 weeks.

Methods and Procedures

- Strongly positive acetylcholine receptor antibody levels

- Senior neurologist opinion

- Improvement following myasthenia gravis treatment and minimal symptoms on discharge

\section{DISCUSSION}

Myasthenia gravis is the most common disorder of the neuromuscular junction, with an annual incidence of 0.21-2 patients per 100,000 ${ }^{[1]}$. It is known that the peak incidence of myasthenia gravis in men occurs at 65 years of age, whereas the peak incidence for women is at 30 years. Late-onset myasthenia gravis often presents with bulbar symptoms, including dysphagia, dysphonia, tongue weakness, slurred speech, and chewing problems ${ }^{[2]}$. This represents a total of $30 \%$ of all myasthenia gravis cases. It is also known that elevated anti-acetylcholine receptor antibody serum titres are the definitive diagnostic test. However, these may also be absent in an older patient set, known as seronegative myasthenia gravis, who have neither antibodies to acetylcholine receptors nor muscle-specific tyrosine kinase ${ }^{[3]}$.

The mainstay of treatment for myasthenia gravis is pyridostigmine, with adjunctive corticosteroids for all patients with myasthenia gravis who have not reached treatment goals following a trial of pyridostigmine. If steroids are not tolerated by the patient, a non-steroidal immunosuppressant can be used, such as cyclosporine, azathioprine or methotrexate. If refractory to these measures, chronic IVIG should be initiated in a speciality centre for myasthenia gravis ${ }^{[4]}$. Some $15-20 \%$ of patients with myasthenia gravis will be affected by myasthenic crisis at least once in their lives and, in one-fifth of patients, this may be their initial presentation ${ }^{[1]}$. The mortality rate in myasthenia crisis has decreased from $42 \%$ in the early 1960 s to $4 \%$ currently ${ }^{[1]}$. Of utmost importance in the treatment of myasthenic crisis is adequate management of respiratory function. Some $66-90 \%$ of patients will require intubation and mechanical ventilation. Non-invasive ventilation can be successfully used to support patients with myasthenic crisis. However, an independent indication of failed non-invasive ventilation is a PCO2 level of $>45 \mathrm{mmHg}$, thus requiring invasive ventilation to be initiated.

It is clear that elective intubation is favourable over an emergent procedure, and this should be anticipated in the ongoing care for a patient in myasthenic crisis. Furthermore, prolonged intubation should be avoided as this can result in further complications, such as increased hospital stay. The most clearly established precipitant of myasthenia crisis is infection. Other precipitating factors are surgery, aspiration pneumonia, pregnancy, and a reduction in immune-modulating medications. Furthermore, certain medications have a clearly established link in precipitating myasthenic crisis, especially corticosteroids. The incidence of corticosteroid-induced myasthenic crisis is understood to be in the range of $9-18 \%{ }^{[3]}$.

Furthermore, this is likely to be increased in older patients with isolated bulbar symptoms. The increased risk of myasthenic crisis following corticosteroid treatment of myasthenia gravis should prompt the initial treatment to be delivered solely in the hospital setting where respiratory function can be monitored and managed appropriately to decrease the risk of increased mortality in these patients.

The initial decline following corticosteroid treatment is not however an indicator of overall response to corticosteroids. As previously mentioned, vigilance should be exercised when dealing with late-onset, isolated bulbar myasthenia gravis and the possibility of progression to myasthenic crisis following initiation of corticosteroids. 


\section{REFERENCES}

1. Wendell L, Levine J. Myasthenic crisis. Neurohospitalist 2011;1:16-22.

2. Basiri K, Ansari B, Okhovat A. Life-threatening misdiagnosis of bulbar onset myasthenia gravis as a motor neuron disease: how much can one rely on exaggerated deep tendon reflexes. Adv Biomed Res 2015;4:58.

3. Montero-Odasso M. Dysphonia as first symptom of late-onset myasthenia gravis. J Gen Intern Med 2006;21:C4-C6.

4. Sanders D, Wolfe G, Narayanaswami P. Author response: International consensus guidance for management of myasthenia gravis: executive summary. Neurology 2017;88:505506. 